\title{
Review Article \\ Recent Research Trends and Future Prospects in Nanozymes
}

\author{
Ho Yun Shin, ${ }^{1}$ Tae Jung Park, ${ }^{2}$ and Moon Il Kim ${ }^{1}$ \\ ${ }^{1}$ Department of BioNano Technology, Gachon University, 1342 Seongnamdae-ro, Sujeong-gu, Seongnam, \\ Gyeonggi 461-701, Republic of Korea \\ ${ }^{2}$ Department of Chemistry, Chung-Ang University, 84 Heukseok-ro, Dongjak-gu, Seoul 156-756, Republic of Korea \\ Correspondence should be addressed to Tae Jung Park; tjpark@cau.ac.kr and Moon Il Kim; moonil@gachon.ac.kr
}

Received 21 July 2015; Accepted 17 November 2015

Academic Editor: Xuping Sun

Copyright (C) 2015 Ho Yun Shin et al. This is an open access article distributed under the Creative Commons Attribution License, which permits unrestricted use, distribution, and reproduction in any medium, provided the original work is properly cited.

\begin{abstract}
Recently, nanomaterial-based enzyme mimetics (nanozymes) have attracted enormous interest. They exhibit unique advantages such as excellent robustness, stability, and low-cost production with easy scale-up, which are critically needed as an alternative to natural enzymes. These nanozymes exhibit natural enzyme-like activity and have been applied to various kinds of detection and treatment methods for biomolecules such as DNA, proteins, cells, and small molecules including glucose. To highlight progress in the field of nanozymes, this review discusses recent nanozyme-based research results and their applications for the development of novel biosensor, immunoassay, cancer diagnostics, therapeutics, and environmental engineering technologies. Current challenges and future prospects of nanozymes for widespread use in biotechnology are also discussed.
\end{abstract}

\section{Introduction}

Early diagnosis of diseases is an area of growing importance for the medical community. The early detection of diseases helps improve therapeutic decision-making, which decreases the severity of illness and length of hospital stay. Accordingly, a number of biosensing techniques have been developed for rapid, reliable, and sensitive detection of biomolecules that can be used as indicators of disease. Among various biosensing methods for diagnosing human diseases, natural enzymes such as horseradish peroxidases have been frequently used for bioassay, as they can catalyze various colorimetric reactions in the presence of specially designed substrates, and they display good sensitivity and selectivity towards the target molecules [1]. In spite of their novel catalytic efficiency, natural enzymes have critical limitations for industrial application, such as low stability in harsh conditions (temperature and $\mathrm{pH}$ ) and relatively high costs for preparation, purification, and storage. Therefore, over the past few decades, researchers have made an intense effort to develop artificial enzymes for a wide range of applications. For example, chemical complexes based on cyclodextrin [2], porphyrin $[3,4]$, hemin $[5,6]$, hematin [7], and specially designed biomolecules in the form of nucleic acids and proteins have been successfully used to mimic the catalytic activity of natural enzymes [8].

In this regard, $\mathrm{Fe}_{3} \mathrm{O}_{4}$ magnetic nanoparticles (MNPs) have been found to exhibit intrinsic peroxidase activity [9]. This remarkable discovery opens up the way for a new class of enzyme mimetics. To date, various nanostructured materials have been reported to possess intrinsic enzymatic activity, including $\mathrm{Fe}_{3} \mathrm{O}_{4}$ magnetic nanoparticles (MNPs) [9], platinum nanoparticles (Pt NPs) [10-12], cerium oxide nanoparticles $\left(\mathrm{CeO}_{2} \mathrm{NPs}\right)$ [13], gold nanoparticles (Au NPs) [14-16], copper oxide nanoparticles (CuO NPs) [17], $\mathrm{BiFeO}_{3}$ nanoparticles [18], $\mathrm{CoFe}_{2} \mathrm{O}_{4}$ nanoparticles [19-21], $\mathrm{FeS}$ and FeSe nanoparticles [22], graphene oxide [23], single-wall carbon nanotubes [24], and hemin-graphene hybrid nanosheets [25]. Herein, we name these nanomaterials which have enzymelike catalytic activity "nanozymes." In contrast to natural enzymes, nanozymes are inherently robust, stable in harsh conditions ( $\mathrm{pH}$ and temperature), and easy to mass-produce with simple scale-up. These advantages make them promising candidates for analytical and environmental applications [26].

In this paper, we present a comprehensive review of recent research on nanozymes and their applications categorized by representative fields of application, such as biosensor, 


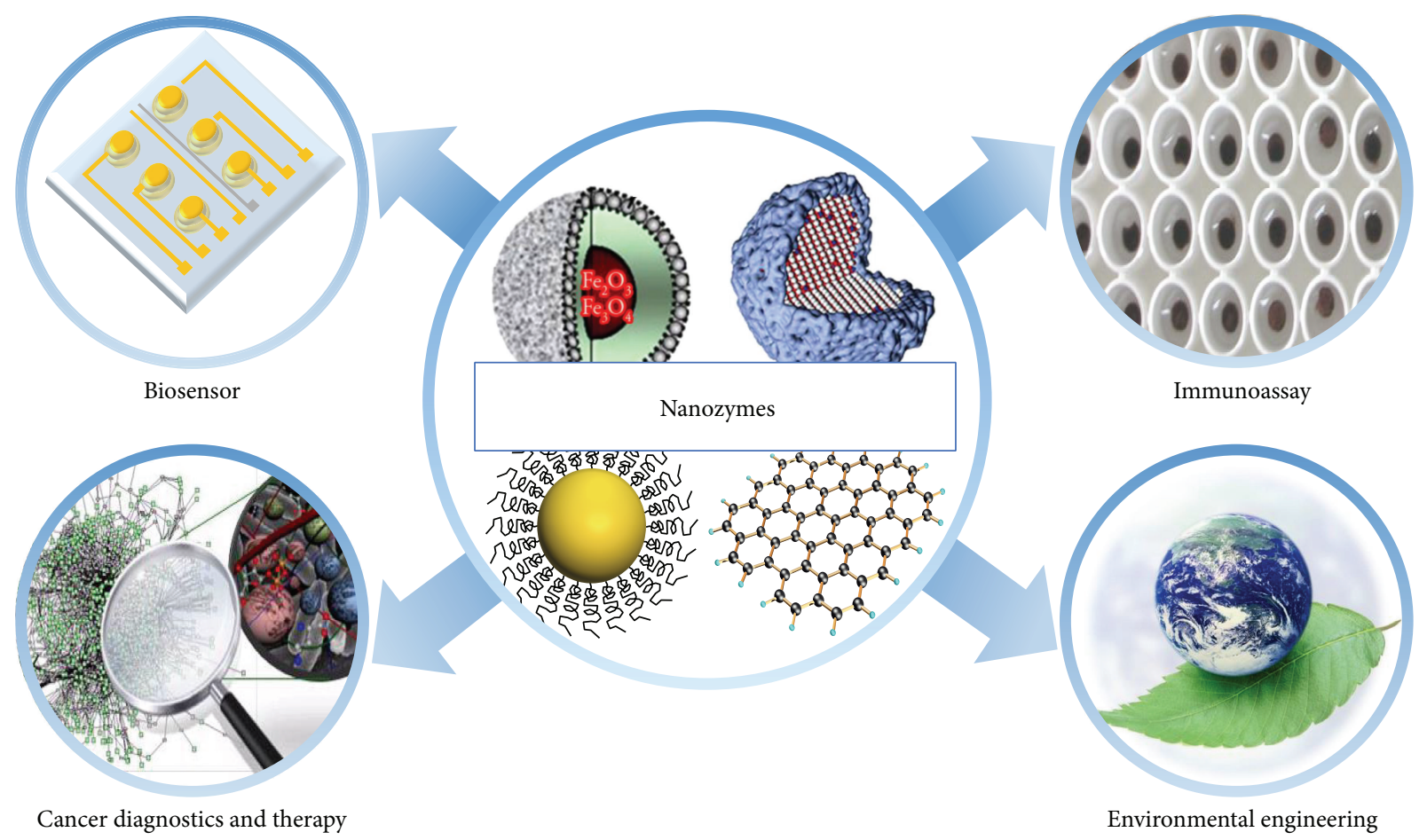

FIGURE 1: A wide range of applications in the field of nanozymes. The images of nanomaterials in the center ring represent (clockwise from top left) $\mathrm{Fe}_{3} \mathrm{O}_{4}$ nanoparticle, $\mathrm{CeO}_{2}$ nanoparticle, graphene oxide, and Au nanoparticle, which are the typical nanomaterials exhibiting enzyme-like activities.

immunoassay, cancer diagnostics, therapeutics, and environmental engineering. We also provide challenges and research trends in the emerging nanozyme research field (Figure 1).

\section{Fundamentals of Nanozymes}

Due to the absence of an active site in nanozymes, where only a specific substrate molecule binds and undergoes a chemical reaction, researchers have developed various strategies to endow nanozymes with specificity to target molecules. The most representative strategies can be divided between the oxidase-coupled method and the surface-modification method. In the oxidase-coupled method, nanozymes with peroxidase-like activity gain their specificity by being coupled with oxidase, which generates $\mathrm{H}_{2} \mathrm{O}_{2}$ as a product of a catalytic reaction which occurs only in the presence of the target molecule. Peroxidase-like nanozymes subsequently catalyze the oxidation of colorimetric substrates with the resulting $\mathrm{H}_{2} \mathrm{O}_{2}$. For the surface-modification method, an antibody is generally conjugated on the surface of the nanozyme to provide specificity toward antigen molecules, mostly in the colorimetric immunoassay system. By conjugation of the antibody specific to the target antigen on the surface of the nanozyme, the nanozyme can act as a target-specific probe, generating a colorimetric signal in the presence of the colorimetric substrate and $\mathrm{H}_{2} \mathrm{O}_{2}$. In the same manner, ligandconjugated nanozymes can specifically bind to target receptors and produce a colorimetric signal when the targeted molecules bind on the nanozymes' surface. Aptamers are attracting interest in the fields of therapeutics and diagnostics and are becoming promising candidates for use in giving specificity to nanozyme-based biosensors.

The intrinsic enzyme-like activities of nanozymes are generally believed to be produced by atoms present on the surface as well as in the nanozyme's inside core. Thus, the atomic composition of nanozymes is the most important factor in determining their catalytic activity, although other factors such as size, morphology, surface coating and modification, $\mathrm{pH}$, and temperature can also have an impact [8]. Based on the type of composition, nanozymes can be distributed into three categories: metal oxide-based, metalbased, and carbon-based nanozymes.

2.1. Metal Oxide-Based Nanozymes. Metal oxide nanoparticles have been widely used in the field of biomedical applications such as biosensor, targeted drug delivery, tissue repair, immunoassay, and contrast agents in magnetic resonance imaging (MRI) and cell separation [27]. Since metal oxide nanoparticles are commonly considered chemically and biologically inert, additional surface engineering and subsequent conjugation with functional substances are required to endow metal oxide nanoparticles with functionality. Recently, inspired by the notable discovery of the intrinsic catalytic activity of MNP as a peroxidase [9], metal oxidetype nanozymes have attracted great interest, with a large number of papers discussing newly reported enzyme-like activities of these nanomaterials. A variety of metal oxidebased nanozymes have been discovered to possess enzymelike catalytic activities (peroxidase, catalase, and superoxide dismutase (SOD)), including MNPs [9], $\mathrm{CeO}_{2}$ NPs [13], 
cobalt oxide nanoparticles $\left(\mathrm{Co}_{3} \mathrm{O}_{4} \mathrm{NPs}\right)$ [28], manganese dioxide nanoparticles $\left(\mathrm{MnO}_{2} \mathrm{NPs}\right)$ [29], vanadium pentoxide nanoparticles $\left(\mathrm{V}_{2} \mathrm{O}_{5} \mathrm{NPs}\right)$ [30], and CuO NPs [31]. Generally, metal oxide-based nanozymes with peroxidase-like activity have been those most widely investigated by researchers, owing to their convenience for constructing amperometric and colorimetric detection systems by utilizing the capability of peroxidase to catalyze certain substrates which generate the corresponding electric and colorimetric signal in the presence of hydrogen peroxide $\left(\mathrm{H}_{2} \mathrm{O}_{2}\right)$. Herein, we focus on colorimetric detection systems utilizing peroxidase-like nanozymes and associated applications.

2.2. Metal-Based Nanozymes. Metal-based nanozymes, such as Au NPs and Pt NPs, have been discovered to possess the catalytic activities of oxidase, peroxidase, catalase, and SOD. In addition to the single nanoparticle system mentioned above, nanocomposites which combine a metalbased nanozyme with other nanozymes have been also intensively developed, including $\mathrm{Fe}_{3} \mathrm{O}_{4}$-graphene oxide (GO) [32], $\mathrm{Fe}_{3} \mathrm{O}_{4}-\mathrm{Pt}$ [33], $\mathrm{Au}-\mathrm{Pt}$ [34], and $\mathrm{GO}-\mathrm{Fe}_{3} \mathrm{O}_{4}-\mathrm{Pt}$ [35] nanocomposites. Surprisingly, it was reported that metalbased nanozymes often exhibit synergistic effects which significantly enhance catalytic performance when coupled with other nanozymes as a composite [33].

2.3. Carbon-Based Nanozymes. Carbon-based nanozymes, such as fullerene, carbon nanotube, graphene oxide, and carbon dot, are also attracting great interest owing to their unique enzyme-mimicking activities [23-25, 36-43]. They have been found to possess peroxidase and SOD-mimicking abilities and are widely utilized as signaling agents for signal amplification and detection of analytes in the field of biosensors and immunoassays.

\section{Recent Applications}

3.1. Biosensors. In recent time, horseradish peroxidase (HRP), which can catalyze the oxidation of a variety of substrates by $\mathrm{H}_{2} \mathrm{O}_{2}$, has been one of the most commonly used enzymes for the construction of biosensors. In spite of its high catalytic efficiency, HRP-based biosensors have critical problems, in that the catalytic activity of HRP is prone to degradation in long-term storage and harsh conditions, thereby leading to errors in the process of sensing. To solve this problem, a peroxidase-like nanozyme which is highly robust against environmental conditions is utilized in place of HRP, providing a cost-effective method of fabrication of biosensors.

3.1.1. $\mathrm{H}_{2} \mathrm{O}_{2}$ Detection. $\mathrm{H}_{2} \mathrm{O}_{2}$ detection plays an essential role in the fields of biology, medicine, environmental engineering, and food industry. Since $\mathrm{H}_{2} \mathrm{O}_{2}$, a product of an incompletely reduced metabolite of oxygen, is generated as a by-product of various biological pathways and is a contaminant in several industrial products and wastes, $\mathrm{H}_{2} \mathrm{O}_{2}$ detection is of practical importance in the field of biosensors. With substantial progress in biosensing technology, various methods for $\mathrm{H}_{2} \mathrm{O}_{2}$ detection have been reported. Among them is the colorimetric signal readout strategy, based on a redox reaction between HRP and colorimetric substrates such as $2,2^{\prime}$ azino-bis(3-ethylbenzothiazoline-6-sulphonic acid) (ABTS) and $3,3^{\prime}, 5,5^{\prime}$-tetramethylbenzidine (TMB). This strategy has been actively developed due to its high sensitivity, selectivity, and simplicity for detecting $\mathrm{H}_{2} \mathrm{O}_{2}$. However, since the colorimetric detection method using HRP has problems of low stability and high cost, nanozymes showing peroxidaselike activity have been intensively utilized to resolve these drawbacks. Beginning with the remarkable discovery that MNPs exhibit intrinsic peroxidase-like activity [9], many researchers have focused on the development of novel $\mathrm{H}_{2} \mathrm{O}_{2}$ detection methods employing various nanozymes. Here, we discuss recent applications of various nanozymes in $\mathrm{H}_{2} \mathrm{O}_{2}$ detection.

After Yan's report, $\mathrm{H}_{2} \mathrm{O}_{2}$ detection methods with MNPs (used as peroxidase-like nanozymes) were further developed by Wei and Wang [44]. This research outlined a colorimetric detection method for $\mathrm{H}_{2} \mathrm{O}_{2}$, in which MNPs were used to catalyze the oxidation of ABTS in the presence of $\mathrm{H}_{2} \mathrm{O}_{2}$. The catalytic oxidation of ABTS with $\mathrm{H}_{2} \mathrm{O}_{2}$ generates greencolored products, by which $\mathrm{H}_{2} \mathrm{O}_{2}$ can be detected by the naked eye. They noted that ABTS could be oxidized by $\mathrm{H}_{2} \mathrm{O}_{2}$ in the absence of any catalysts but demonstrated that the presence of MNPs gave a 320\% higher response of absorption spectra when compared with the absence of MNPs. Not only the peroxidase-like catalytic activity of $\mathrm{Fe}_{3} \mathrm{O}_{4}$ MNPs but also their high stability in rough conditions ( $\mathrm{pH}$ and temperature) was confirmed with additional investigations.

Based on the colorimetric detection method above, many approaches have proceeded by varying the colorimetric substrates and nanozymes used. N,N-Dimethyl- $p$ phenylenediamine sulfate (DPD) was used as a colorimetric substrate in place of ABTS [45]. The DPD-MNPs analytical system showed several advantages (lower operating temperature and detection limit and higher sensitivity) over the system using ABTS, because DPD is more easily oxidized by $\mathrm{H}_{2} \mathrm{O}_{2}$ than ABTS and because oxidized DPD (DPD ${ }^{+}$) produces a colored product with a strong absorption maximum at $550 \mathrm{~nm}$. Aside from the signal-on colorimetric method above, Jiang's group has developed a new type of fluorescence method using rhodamine $\mathrm{B}(\mathrm{RhB})$ as a substrate [46]. In this method, MNPs catalyze $\mathrm{H}_{2} \mathrm{O}_{2}$ to form the radical $\mathrm{OH}$, which can oxidize $\mathrm{RhB}$ to form colorless and nonfluorescent products. In short, the more the $\mathrm{H}_{2} \mathrm{O}_{2}$ exists, the weaker the fluorescence intensity of $\mathrm{RhB}$ is.

Other nanozymes have been also reported to detect $\mathrm{H}_{2} \mathrm{O}_{2}$. Au NPs have been discovered to possess intrinsic peroxidase-like activity [47]. Cao's studies reported that positively charged $\mathrm{Au}$ NPs can catalyze the oxidation of TMB by $\mathrm{H}_{2} \mathrm{O}_{2}$. Carboxyl-modified graphene oxide was shown to possess intrinsic peroxidase-like activity that can catalyze the reaction of TMB in the presence of $\mathrm{H}_{2} \mathrm{O}_{2}$ to produce a bluecolor reaction [23]. Concentrations as low as $5 \times 10^{-8} \mathrm{~mol} \mathrm{~L}^{-1}$ $\mathrm{H}_{2} \mathrm{O}_{2}$ were detected with a linear range from $5 \times 10^{-8}$ to $1 \times$ $10^{-6} \mathrm{~mol} \mathrm{~L}^{-1}$.

3.1.2. Glucose Detection. Peroxidase-like nanozymes coupled with glucose oxidase (GOx) have been frequently employed 
in the construction of glucose biosensors. Wang et al. developed a colorimetric glucose detection platform by combining the catalytic oxidation of glucose with GOx and the catalytic reaction of ABTS with MNPs [48]. Glucose concentrations as low as $30 \mu \mathrm{M}$ were detected with a linear range from $50 \mu \mathrm{M}$ to $1 \mathrm{mM}$ in this study.

There have been several reports of an electrochemical biosensing platform using GOx-coupled nanozymes. A highly efficient and robust electrochemical biosensing strategy employing a nanocomposite harboring GOx-coupled nanozymes was developed by our group [49]. In this report, MNPs and GOx were entrapped in the pores of mesoporous carbon, in which GOx immobilized in the nanocomposite generates $\mathrm{H}_{2} \mathrm{O}_{2}$ which then is directly reduced to $\mathrm{H}_{2} \mathrm{O}$, with the electrocatalytic reduction mediated by MNPs. This system showed a linear range of $(0.5$ to 10$) \times 10^{-3} \mathrm{M}$ and a detection limit of $0.2 \times 10^{-3} \mathrm{M}$.

3.1.3. Oxidase-Coupled Methods (Except Glucose Oxidase). On the basis of the mechanism of glucose detection above, several different oxidases have also been coupled with nanozymes for the fabrication of biosensing platforms. A nanostructured multicatalyst system consisting of MNPs and cholesterol oxidase entrapped in large-pore-sized mesoporous silica has been developed for convenient colorimetric detection of cholesterol [50]. This multicatalyst system is composed of MNPs incorporated in the wall of mesocellular silica pores, forming magnetic mesoporous silica (MMS), and cholesterol oxidases. In this system, cholesterol oxidase immobilized in the MMS promotes a reaction with cholesterol to generate $\mathrm{H}_{2} \mathrm{O}_{2}$, which subsequently activates MNPs in the mesocellular silica pores to convert a colorimetric substrate into a colored product. The result of this investigation shows the cholesterol oxidase-coupled method to have high selectivity and sensitivity (limit of detection, LOD, of $5 \mu \mathrm{M}$ in the linear range from 10 to $250 \mu \mathrm{M}$ ) for the detection of cholesterol. A colorimetric method for detection of galactose, which utilizes a nanostructured multicatalyst system consisting of MNPs and galactose oxidase, has also been reported [51]. The clinical applicability of this multicatalytic system was successfully evaluated as a promising analytical tool to diagnose galactosemia, by determining the concentration of galactose eluted from the dried blood specimens provided by clinical hospitals.

Apart from the above oxidases, alcohol oxidase has been used in a colorimetric biosensor for quantification of ethanol and methanol [52]. The nanocomposite system utilizing alcohol oxidase entrapped in mesocellular silica with MNPs provided a rapid and convenient platform for analysis of alcohol, with high stability and reusability. It showed a linear concentration range from 100 to $500 \mu \mathrm{M}$ with a detection limit as low as $25 \mu \mathrm{M}$.

3.1.4. Other Methods. Recently, peroxidase-mimicking nanomaterials such as MNPs, $\mathrm{CeO}_{2}$ NPs, and Au NPs [15] have been employed for new methods of DNA detection. A labelfree colorimetric detection method for nucleic acids has been developed [53]. In this method, the target DNA in the sample, which is amplified by polymerase chain reaction
(PCR), is directly adsorbed on the surface of the MNPs due to electrostatic interactions between the negatively charged phosphate backbone and the positively charged surface of the NPs, thereby inducing a shielding effect against colorimetric substrate binding to MNPs. The peroxidase activity of MNPs will decrease due to the DNA-induced shielding, so that the intensity of the color signal will also be significantly reduced. Using this detection method, researchers successively detected Chlamydia trachomatis in human urine, which is one of the common bacteria causing sexually transmitted disease (STD) $[54,55] . \mathrm{CeO}_{2}$ NPs were also employed in a label-free colorimetric method for detecting C. trachomatis, and this method provided ultrafast detection of the target nucleic acid (target nucleic acids can be determined within a few minutes) [56].

A novel biosensing format using aptamers has been developed by several researchers based on the fact that aptamers, which are ssDNA or ssRNA that can specifically bind to a target, can replace antibodies for the specific recognition of target molecules. A method using chitosan-modified MNPs conjugated with thrombin aptamers was reported by Zhang's group [57]. They constructed a sandwich-type assay for the detection of thrombin with two thrombin aptamers. This aptamer-based assay showed a linear detection range from 1 to $100 \mathrm{nM}$ and a detection limit of $1 \mathrm{nM}$ of thrombin.

3.2. Immunoassays. Immunoassays have been used in hospitals, laboratory, medicine, and research to improve the health and well-being of humans and animals. Information gained by clinical immunoassay testing has shortened the length of hospital stays and decreased the severity of illness by identifying and assessing the progression of disease, thereby leading to improved therapeutic choices. In life science research, immunoassays are used in the study of biological systems for tracking different proteins, hormones, and antibodies. In industry, immunoassays are used to detect contaminants in food and water and in quality control to monitor specific molecules used during product processing. However, the most commonly used enzymes in immunoassay include horseradish peroxidase and alkaline phosphatase [58-62], which lose their enzymatic activities gradually over long-term storage [63]. To overcome these limits, various studies on replacing natural enzymes have been reported, and consequently novel types of immunoassay using nanozymes in place of HRP have been developed.

3.2.1. Sandwich or Antigen-Down Type Immunoassays. Gao and coworkers reported an immunoassay using chitosanmodified MNPs (CS-MNPs) as a replacement for HRP in the traditional immunoassay [63]. They provided protocols for antigen-down and sandwich immunoassays with CS-MNPs and detected mouse IgG and carcinoembryonic antigen (CEA), respectively. Chitosan modified on the surface of MNPs prevented aggregation of MNPs, so that MNPs were easily dispersed in aqueous solutions. Meanwhile, amino groups in the chitosan provided a convenient site for covalent linking of antibodies to MNPs, thereby replacing the linkage of HRP-conjugated antibodies to CS-MNP-conjugated antibodies in the immunoassay. Capture-detection immunoassay 
was also developed to detect CEA by employing the magnetic properties of CS-MNPs, which facilitate capturing, separating, and enriching antigens as well as redispersing the MNP aggregation in solution.

Zhang and coworkers reported a novel immunoassay utilizing Prussian blue modified $\gamma-\mathrm{Fe}_{2} \mathrm{O}_{3}$ NPs [64]. Prussian blue, a dark blue pigment with the idealized formula $\mathrm{Fe}_{7}(\mathrm{CN})_{18}$, was modified on the surface of $\gamma-\mathrm{Fe}_{2} \mathrm{O}_{3}$ NPs due to its excellent electrochemical behavior that accelerated electron transfer and its catalytic properties that could catalyze the reduction of $\mathrm{H}_{2} \mathrm{O}_{2}$. Prussian blue modified $\gamma-\mathrm{Fe}_{2} \mathrm{O}_{3} \mathrm{NPs}$ (PBMNPs) were next conjugated with staphylococcal protein A (SPA) to bind to IgG immobilized in the well, so that PBMNPs could derive a colorimetric reaction in the presence of TMB and $\mathrm{H}_{2} \mathrm{O}_{2}$.

Ferritins, nanoscale globular protein cages encapsulating a ferric core, were used in immunoassay in Tang et al.'s study [65]. In this study, ferritin showed a thermally stable and $\mathrm{pH}$ tolerable enzyme-mimetic activity derived from the ferric nanocore of ferritin. Two forms of immunoassay systems were constructed: antigen-down type and sandwich type. Avidin was selected as the target molecule of the antigendown immunoassay and nitrated human ceruloplasmin as the target molecule of the sandwich-type immunoassay. They also reported that the ferritins could be utilized in analytical applications such as $\mathrm{H}_{2} \mathrm{O}_{2}$ assay. In their assay, ferritin oxidizes the p-HPPA in the presence of $\mathrm{H}_{2} \mathrm{O}_{2}$ to generate a fluorescent product. This ferritin-based $\mathrm{H}_{2} \mathrm{O}_{2}$ assay shows a detection limit of $0.16 \mu \mathrm{M}$ and a linear detection range of $40 \mu \mathrm{M}$, which is one-order higher sensitivity with a broader linear response range.

Immunoassay systems for detection of rotavirus and breast cancer have been developed [66], in which MNPs are conjugated to antibodies against rotaviruses and human epidermal growth factor receptor 2 (HER2). In this system, sandwich-type and antigen-down type immunoassay were used to detect rotavirus and HER2, respectively. For the detection of rotavirus, rotavirus antibodies were first immobilized in a well and rotavirus bound to the immobilized antibody. Subsequently, MNP-conjugated antibodies (MNP-Abs) were added to the well in order to bind to captured rotaviruses. Finally, the peroxidase substrate TMB was changed into blue-colored products in the presence of $\mathrm{H}_{2} \mathrm{O}_{2}$. An antigen-down immunoassay system was used in the case of breast cell detection, which did not require prior immobilization of antibodies in the bare well surface. Breast cells were cultured in a well so that they adsorbed to the surface of the well. MNPs-Abs were then applied to the cellcultured well, followed by adding TMB and $\mathrm{H}_{2} \mathrm{O}_{2}$ to induce a colorimetric reaction. This assay system displayed excellent specificity, sensitivity, and linearity for quantitative detection of the target molecules, as well as the production of a color signal that could be detected by the naked eye.

Based on the above system, a nanocomposite-based immunoassay was also performed, in which nanocomposite entrapping MNPs and Pt NPs in ordered mesoporous carbon (OMC) were utilized instead of HRP [33]. This immunoassay generated significantly higher absorption intensity of color signal than the current ELISA and was able to quantify the target antigen very rapidly within three minutes, while the conventional ELISA requires several tens of minutes for color signal development $[67,68]$. It showed a limit of detection (LOD) for HER2 of $1.5 \mathrm{ng} \mathrm{mL}^{-1}$ in the linear range from 2.5 to $100 \mathrm{ng} \mathrm{mL}^{-1}$. The nanocomposite was found to have 50 times higher catalytic efficiency than that of free MNPs, owing to the high catalytic action of Pt NPs.

Graphene oxide (GO) has been used in immunoassay as a peroxidase-mimicking nanozyme [41]. Yan's group developed a sandwich-type immunoassay for the detection of cancer biomarker prostate specific antigen (PSA). In this work, a magnetic bead (MB) was used to immobilize the primary PSA antibody $\left(\mathrm{Ab}_{1}\right)$ and then a GO-conjugated secondary antibody $\left(\mathrm{Ab}_{2}\right)$ was applied in the presence of PSA. Subsequently, $M B-A b_{1}$ was separated from the immunocomplex by an external magnetic field, and GO catalyzed the oxidation of hydroquinone in the presence of $\mathrm{H}_{2} \mathrm{O}_{2}$ to generate a browncolored product.

Conjugating both MNPs and Pt NPs on the surface of GO enabled highly sensitive and rapid colorimetric detection of the target cancer cell [35]. In this work, it was notable that the electron transfer between MNPs and Pt NPs creates a synergistic effect, significantly enhancing the catalytic performance of MNPs-Pt NPs-GO nanohybrids. Using this immunoassay system, human breast adenocarcinoma cells (SKBR-3), which overexpressed HER2, were detected in five minutes with high specificity and sensitivity. The LOD for target SKBR-3 cells was found to be about 100 cells in the linear range from 100 to 1000 cells. Moreover, fluorescence imaging of SKBR-3 was successfully performed with MNPsPt NPs-GO nanohybrids.

3.2.2. Other Immunoassays. By employing the superparamagnetic property of MNPs, a capture-detection immunoassay system has been developed by Gao's group [63]. In the procedure, the CS-MNPs were conjugated with carcinoembryonic antibodies (anti-CEA M111147) and then mixed with the sample containing CEA. After the CEA was captured by MNPs, a magnetic field was applied to separate the MNPs which had captured CEA. Finally, the MNPs capturing CEA were injected into microplate wells coated with another monoclonal CEA antibody, creating the sandwich format. Thereby, the MNPs prompted the generation of a color signal upon addition of colorimetric substrate and $\mathrm{H}_{2} \mathrm{O}_{2}$ to the wells. Cardiac troponin I ( TnI) in serum, a well-known biomarker for myocardial infarction, was also detected by a capture-detection immunoassay utilizing the magnetism and peroxidase ability of MNPs [9].

3.3. Cancer Diagnostics without Immune Reaction. Aside from immunoassay using antigen-antibody interaction, other novel assays using nanozymes have been developed particularly for the diagnosis of tumor cells. Asati and coworkers reported an assay for the determination of tumor cells with poly(acrylic acid)-coated $\mathrm{CeO}_{2} \mathrm{NPs}$ (nanoceria) as an oxidase mimic [13]. When the nanoparticles were conjugated with folic acid, they bound to folate receptors on the tumor cell (A-549 lung cancer cells), due to high expression of folate receptors on the tumor cell surface. Polymer-coated 
nanoceria as an oxidase mimic made detection of tumor cells easier than with traditional immunoassay, because it directly oxidized a colorimetric substrate to a colored product without $\mathrm{H}_{2} \mathrm{O}_{2}$ and additional steps to introduce an enzymeconjugated secondary antibody. Further advances in this technology were also reported by employing a fluorescencegenerating substrate, ampliflu, to detect target cancer cells at around neutral pH [69].

Another interesting study to visualize target tumor tissues without the use of any additional targeting ligands has also been described [70]. In this study, peroxidase-like iron oxide nanoparticles were encapsulated inside a recombinant human heavy-chain ferritin (Hfn) protein shell, which binds to tumor cells that overexpress transferrin receptor 1 . The iron oxide cores catalyzed the oxidation of peroxidase substrates in the presence of $\mathrm{H}_{2} \mathrm{O}_{2}$ to produce the colorimetric signal that was used to visualize tumor tissues. Through this strategy, nine types of cancer were successfully verified with enough specificity and sensitivity.

3.4. Therapeutic Applications. As described above, nanozymes have been widely used for detection and diagnostic methods. Besides these applications, many researchers have also studied therapeutic applications including antiinflammatory effects, neuroprotection, stem cell growth, and antiaging. In general, SOD was often utilized for therapeutic applications owing to its protective role as a scavenger of reactive oxygen intermediates (ROIs). Intracellular concentration of ROI, including hydrogen peroxidase, hypochlorite ions, hydroxyl radicals, hydroxyl ions, and superoxide anions, has been known to be a cause of cell degeneration and associated diseases [71]. Inspired by earlier studies by Seal et al. which revealed the activity of $\mathrm{CeO}_{2}$ NPs as a SOD-mimic [72, 73], various studies have been attempted to develop SODmimicking nanozymes.

Chen at al. reported that nanoceria as a SOD-mimic prevented retinal degeneration by inhibiting the production of ROIs [74]. In their work, nanoceria prevented ROIinduced apoptosis and intracellular accumulation of ROI in cultured retinal neurons in the presence of $\mathrm{H}_{2} \mathrm{O}_{2}$. They further demonstrated that nanoceria injected into the eyes of rats protected retina photoreceptor cells from light-induced degeneration. The study by Hirst et al. also demonstrated that nanoceria could be used for anti-inflammation by elimination of the radical oxygen species in J774A.1 murine macrophage cells [75].

Superparamagnetic iron oxide (SPIO) nanoparticles have been employed to promote growth of stem cells. Huang et al. reported that Ferucarbotran, a commercialized SPIO, could promote cell growth in human mesenchymal stem cells (hMSCs) by diminishing intracellular $\mathrm{H}_{2} \mathrm{O}_{2}$ and also accelerate cell cycle progression [76]. In this report, the intrinsic peroxidase-like activity of SPIO dramatically reduced intracellular $\mathrm{H}_{2} \mathrm{O}_{2}$ after internalization into hMSCs, as well as free iron ions released from lysosomal degradation of SPIOaffected cell cycle control molecules.

3.5. Environmental Engineering. Recently, environmental problems such as water and air pollution, food safety, and public health have become growing concerns in society. In addition to the aforementioned applications, nanozymebased techniques have been explored for use in the field of environmental technology.

3.5.1. Pollutant Detection. Ding et al. developed a simple and rapid colorimetric method for detecting melamine, an organic nitrogenous compound which is toxic when swallowed and has been illegally added to dairy products [77]. The principle of this method is as follows. Melamine inhibits the catalytic oxidation of colorimetric substrates (ABTS) by MNPs in the presence of $\mathrm{H}_{2} \mathrm{O}_{2}$, because it competitively reacts with $\mathrm{H}_{2} \mathrm{O}_{2}$, forming an additional compound. Consequently, the intensity of the ABTS color signal was dependent on the concentration of melamine. On the basis of this reaction, a colorimetric system using MNPs could enable easy detection by the naked eye of concentrations of melamine above safety limits in dairy products.

Nanocomposite-entrapping MNPs and oxidase in mesoporous carbon were used to detect several phenol compounds amperometrically, such as phenol, cresol, and cathechol [49]. These phenol compounds produced a concentrationdependent increase of cathodic current in this system, which may have great potential in the field of environmental monitoring.

3.5.2. Pollutant Removal. Although there have been many methods for removal of industrial dyestuffs, such as absorption, precipitation, and ultrasonic decomposition, they could not efficiently degrade organic pollutants in wastewater. Nanozyme-based methods have been found to be a powerful, cost-effective, and simple method for degradation and mineralization of organic dyes from industrial processes. Most prominently, MNPs such as peroxidase have been investigated for degradation of organic pollutants, such as methylene blue, phenol, and rhodamine B. A MNP-based degradation method offers distinct advantages over existing degradation methods which use HRP, such as lower cost, high stability, and reusability. MNPs- $\mathrm{H}_{2} \mathrm{O}_{2}$ could remove $85 \%$ of phenol from aqueous solution within three hours [78]. The MNPs-based degradation showed higher efficiency compared to HRP-based degradation and stability in a broad range of temperatures $\left(5-90^{\circ} \mathrm{C}\right)$, leading to ease of storage. Furthermore, MNPs could be captured by the application of an external field and recycled for five rounds, retaining almost $100 \%$ of their activity. Removal of methylene blue by MNPs$\mathrm{H}_{2} \mathrm{O}_{2}$ coupled method has also been successfully performed by Jiang et al. [79]. It was observed that $96 \%$ of methylene blue was degraded in 15 minutes at optimized condition.

$\mathrm{GaO}$ et al. reported that $\mathrm{MNPs}-\mathrm{H}_{2} \mathrm{O}_{2}$ system could degrade biofilm and kill resident bacteria [80]. Biofilm, especially formed by Pseudomonas aeruginosa, occurs in hospital water systems and medical devices with high frequency, becoming a common cause of nosocomial infection [81]. In this report, MNPs- $\mathrm{H}_{2} \mathrm{O}_{2}$ system exhibited significantly higher efficiency than the use of $\mathrm{H}_{2} \mathrm{O}_{2}$ in degradation of biofilm. The authors confirmed that additional free hydroxyl radicals generated by MNP catalysis of $\mathrm{H}_{2} \mathrm{O}_{2}$ facilitated the oxidative cleavage of biofilm components (nucleic acids, 


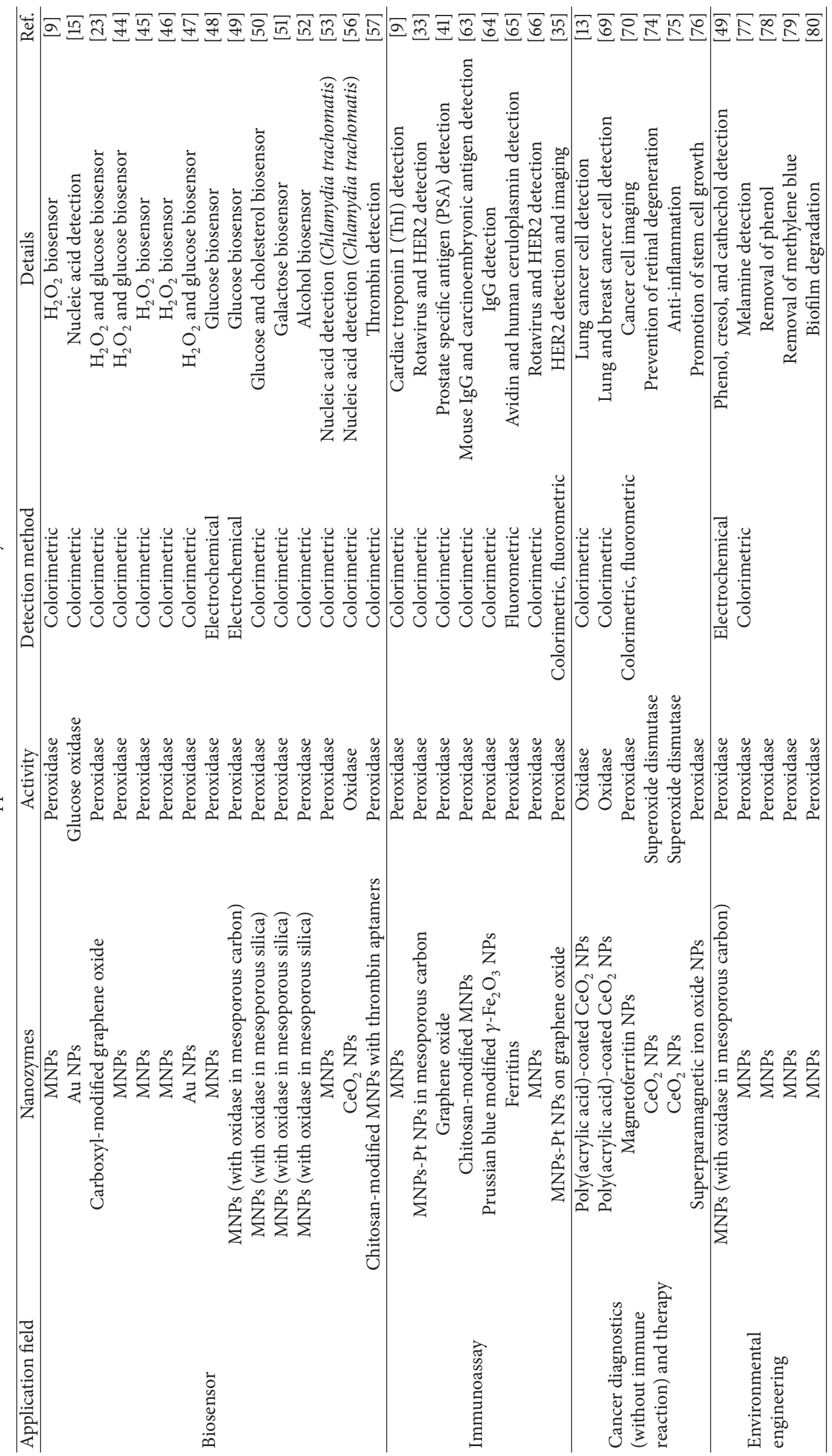


proteins, and polysaccharides) as well as killing resident bacteria.

\section{Conclusions and Future Research Aspects}

Nanozymes have recently emerged as a potent alternative to natural enzymes. As discussed above, although they are still in the initial stages of research, their use has developed substantially in many different detection and treatment methods for biomolecules (Table 1). Despite the advantages of nanozymes such as their low cost, high stability, robustness, ease of mass production, and long-term storability, there are several challenges to be tackled for practical use. Firstly, most nanozymes have low activity compared to natural enzymes. Even if the nanomaterial itself is highly active, additional coating and surface modification can decrease its performance. Therefore, development of novel nanozymes exhibiting high activity and appropriate surface-modification techniques are the emerging issues in the field of nanozymes. Nanozymes also have low selectivity to targets, owing to the absence of active sites where a substrate molecule binds and undergoes a chemical reaction in a natural enzyme. Although researchers have designed various types of surface-modified nanozymes with polymers, nucleic acids, and antibodies to provide selectivity mimicking natural enzymes, this is still insufficient for use in practical applications. Toxicity of nanozymes to humans and the ecosystem is also an essential issue to be solved in regard to environmental and therapeutic applications.

In order for nanozymes to be positioned as a novel source technology by efficiently overcoming the limitations of natural enzymes, we offer the following suggestions. The development of new nanozymes with higher activity and other positive properties than existing nanozymes is required. While traditional research on developing nanozymes has been performed by random screening of the enzymelike activities of existing unspecified nanomaterials, future research will follow a strategy of rational screening of enzyme-like activity based on those atomic compositions which are envisaged to catalyze enzymatic reactions. Furthermore, a strategy to prepare composites can be expected to resolve the current major limitations of nanozymes of low catalytic activity, by exploiting their synergistic effect to facilitate electron transfer between composite materials during redox reaction. Bioinspired synthesis of nanozymes also provides an option to prepare nontoxic nanozymes, by effectively circumventing the use of toxic chemicals in conventional chemical synthesis, thereby accelerating their use in therapeutic applications. Finally, the development of novel surface engineering technology that can make nanozymes selective to target substrates will be important in this field. With the abovementioned research projects, we expect nanozymes to be widely employed in a wide range of applications in the near future.

\section{Conflict of Interests}

The authors declare no financial or commercial conflict of interests.

\section{Acknowledgments}

This work was supported by the Basic Science Research Program through the National Research Foundation of Korea (NRF) funded by the Ministry of Science, ICT \& Future Planning (NRF-2014R1A1A1006016) and by the Gachon University research fund of 2014 (GCU-2014-0110).

\section{References}

[1] D. L. Nelson and M. M. Cox, Lehninger Principles of Biochemistry, vol. 6, chapter 6, W. H. Freeman, New York, NY, USA, 2005.

[2] Z. Liu, R. Cai, L. Mao, H. Huang, and W. Ma, "Highly sensitive spectrofluorimetric determination of hydrogen peroxide with $\beta$-cyclodextrin-hemin as catalyst," Analyst, vol. 124, no. 2, pp. 173-176, 1999.

[3] R. P. Bonar-Law and J. K. M. Sanders, "Polyol recognition by a steroid-capped porphyrin. Enhancement and modulation of misfit guest binding by added water or methanol," Journal of the American Chemical Society, vol. 117, no. 1, pp. 259-271, 1995.

[4] X.-M. Huang, M. Zhu, L.-Y. Mao, and H.-X. Shen, "Catalytic determination of hydrogen peroxide by using the molybdenum-porphyrin complex as a mimetic enzyme of peroxidase," Analytical Sciences, vol. 13, no. 1, pp. 145-147, 1997.

[5] L. Fruk and C. M. Niemeyer, "Covalent hemin-DNA adducts for generating a novel class of artificial heme enzymes," Angewandte Chemie-International Edition, vol. 44, no. 17, pp. 2603-2606, 2005.

[6] Q. Wang, Z. Yang, X. Zhang, X. Xiao, C. K. Chang, and B. Xu, "A supramolecular-hydrogel-encapsulated hemin as an artificial enzyme to mimic peroxidase," Angewandte Chemie International Edition, vol. 46, no. 23, pp. 4285-4289, 2007.

[7] Z. Genfa and P. K. Dasgupta, "Hematin as a peroxidase substitute in hydrogen peroxide determinations," Analytical Chemistry, vol. 64, no. 5, pp. 517-522, 1992.

[8] H. Wei and E. Wang, "Nanomaterials with enzyme-like characteristics (nanozymes): next-generation artificial enzymes," Chemical Society Reviews, vol. 42, no. 14, pp. 6060-6093, 2013.

[9] L. Gao, J. Zhuang, L. Nie et al., "Intrinsic peroxidase-like activity of ferromagnetic nanoparticles," Nature Nanotechnology, vol. 2, no. 9, pp. 577-583, 2007.

[10] R. Polsky, R. Gill, L. Kaganovsky, and I. Willner, "Nucleic acidfunctionalized Pt nanoparticles: catalytic labels for the amplified electrochemical detection of biomolecules," Analytical Chemistry, vol. 78, no. 7, pp. 2268-2271, 2006.

[11] T. Li, Y. Du, and E. Wang, "Polyethyleneimine-functionalized platinum nanoparticles with high electrochemiluminescence activity and their applications to amplified analysis of biomolecules," Chemistry-An Asian Journal, vol. 3, no. 11, pp. 1942-1948, 2008.

[12] W. W. He, Y. Liu, J. S. Yuan et al., "Au@Pt nanostructures as oxidase and peroxidase mimetics for use in immunoassays," Biomaterials, vol. 32, no. 4, pp. 1139-1147, 2011.

[13] A. Asati, S. Santra, C. Kaittanis, S. Nath, and J. M. Perez, "Oxidase-like activity of polymer-coated cerium oxide nanopartieles," Angewandte Chemie-International Edition, vol. 48, no. 13, pp. 2308-2312, 2009.

[14] W. J. Luo, C. F. Zhu, S. Su et al., "Self-catalyzed, self-limiting growth of glucose oxidase-mimicking gold nanoparticles," ACS Nano, vol. 4, no. 12, pp. 7451-7458, 2010. 
[15] X. Zheng, Q. Liu, C. Jing et al., "Catalytic gold nanoparticles for nanoplasmonic detection of DNA hybridization," Angewandte Chemie International Edition, vol. 50, no. 50, pp. 11994-11998, 2011.

[16] Y. J. Long, Y. F. Li, Y. Liu, J. J. Zheng, J. Tang, and C. J. Huang, "Visual observation of the mercury-stimulated peroxidase mimetic activity of gold nanoparticles," Chemical Communications, vol. 47, no. 43, pp. 11939-11941, 2011.

[17] W. Chen, J. Chen, Y.-B. Feng et al., "Peroxidase-like activity of water-soluble cupric oxide nanoparticles and its analytical application for detection of hydrogen peroxide and glucose," Analyst, vol. 137, no. 7, pp. 1706-1712, 2012.

[18] W. Luo, Y.-S. Li, J. Yuan et al., "Ultrasensitive fluorometric determination of hydrogen peroxide and glucose by using multiferroic $\mathrm{BiFeO}_{3}$ nanoparticles as a catalyst," Talanta, vol. 81, no. 3, pp. 901-907, 2010.

[19] S. H. He, W. B. Shi, X. D. Zhang, J. A. Li, and Y. M. Huang, “ $\beta$ Cyclodextrins-based inclusion complexes of $\mathrm{CoFe}_{2} \mathrm{O}_{4}$ magnetic nanoparticles as catalyst for the luminol chemiluminescence system and their applications in hydrogen peroxide detection," Talanta, vol. 82, no. 1, pp. 377-383, 2010.

[20] W. B. Shi, X. D. Zhang, S. H. He, and Y. M. Huang, " $\mathrm{CoFe}_{2} \mathrm{O}_{4}$ magnetic nanoparticles as a peroxidase mimic mediated chemiluminescence for hydrogen peroxide and glucose," Chemical Communications, vol. 47, no. 38, pp. 10785-10787, 2011.

[21] Y. W. Fan and Y. M. Huang, "The effective peroxidase-like activity of chitosan-functionalized $\mathrm{CoFe}_{2} \mathrm{O}_{4}$ nanoparticles for chemiluminescence sensing of hydrogen peroxide and glucose," Analyst, vol. 137, no. 5, pp. 1225-1231, 2012.

[22] A. K. Dutta, S. K. Maji, D. N. Srivastava et al., "Synthesis of FeS and FeSe nanoparticles from a single source precursor: a study of their photocatalytic activity, peroxidase-like behavior, and electrochemical sensing of $\mathrm{H}_{2} \mathrm{O}_{2}$," ACS Applied Materials and Interfaces, vol. 4, no. 4, pp. 1919-1927, 2012.

[23] Y. Song, K. Qu, C. Zhao, J. Ren, and X. Qu, "Graphene oxide: intrinsic peroxidase catalytic activity and its application to glucose detection," Advanced Materials, vol. 22, no. 19, pp. 22062210, 2010.

[24] Y. Song, X. Wang, C. Zhao, K. Qu, J. Ren, and X. Qu, "Labelfree colorimetric detection of single nucleotide polymorphism by using single-walled carbon nanotube intrinsic peroxidaselike activity," Chemistry - A European Journal, vol. 16, no. 12, pp. 3617-3621, 2010.

[25] Y. Guo, J. Li, and S. Dong, "Hemin functionalized graphene nanosheets-based dual biosensor platforms for hydrogen peroxide and glucose," Sensors and Actuators B: Chemical, vol. 160, no. 1, pp. 295-300, 2011.

[26] J. Xie, X. Zhang, H. Wang, H. Zheng, Y. Huang, and J. Xie, "Analytical and environmental applications of nanoparticles as enzyme mimetics," TrAC-Trends in Analytical Chemistry, vol. 39, pp. 114-129, 2012.

[27] A. K. Gupta and M. Gupta, "Synthesis and surface engineering of iron oxide nanoparticles for biomedical applications," Biomaterials, vol. 26, no. 18, pp. 3995-4021, 2005.

[28] J. Mu, Y. Wang, M. Zhao, and L. Zhang, "Intrinsic peroxidaselike activity and catalase-like activity of $\mathrm{Co}_{3} \mathrm{O}_{4}$ nanoparticles," Chemical Communications, vol. 48, no. 19, pp. 2540-2542, 2012.

[29] Y. Wan, P. Qi, D. Zhang, J. Wu, and Y. Wang, "Manganese oxide nanowire-mediated enzyme-linked immunosorbent assay," Biosensors and Bioelectronics, vol. 33, no. 1, pp. 6974, 2012.
[30] R. André, F. Natálio, M. Humanes et al., " $\mathrm{V}_{2} \mathrm{O}_{5}$ nanowires with an intrinsic peroxidase-like activity," Advanced Functional Materials, vol. 21, no. 3, pp. 501-509, 2011.

[31] W. Chen, J. Chen, A.-L. Liu, L.-M. Wang, G.-W. Li, and X.H. Lin, "Peroxidase-like activity of cupric oxide nanoparticle," ChemCatChem, vol. 3, no. 7, pp. 1151-1154, 2011.

[32] Y.-L. Dong, H.-G. Zhang, Z. U. Rahman et al., "Graphene oxide$\mathrm{Fe}_{3} \mathrm{O}_{4}$ magnetic nanocomposites with peroxidase-like activity for colorimetric detection of glucose," Nanoscale, vol. 4, no. 13, pp. 3969-3976, 2012.

[33] M. I. Kim, Y. Ye, M.-A. Woo, J. Lee, and H. G. Park, "A highly efficient colorimetric immunoassay using a nanocomposite entrapping magnetic and platinum nanoparticles in ordered mesoporous carbon," Advanced Healthcare Materials, vol. 3, no. 1, pp. 36-41, 2014.

[34] J. Liu, X. Hu, S. Hou et al., “Au@Pt core/shell nanorods with peroxidase- and ascorbate oxidase-like activities for improved detection of glucose," Sensors and Actuators B: Chemical, vol. 166-167, pp. 708-714, 2012.

[35] M. I. Kim, M. S. Kim, M.-A. Woo et al., "Highly efficient colorimetric detection of target cancer cells utilizing superior catalytic activity of graphene oxide-magnetic-platinum nanohybrids," Nanoscale, vol. 6, no. 3, pp. 1529-1536, 2014.

[36] M. Liu, H. Zhao, S. Chen, H. Yu, and X. Quan, "Interface engineering catalytic graphene for smart colorimetric biosensing," ACS Nano, vol. 6, no. 4, pp. 3142-3151, 2012.

[37] Y. Ye, T. Kong, X. Yu, Y. Wu, K. Zhang, and X. Wang, "Enhanced nonenzymatic hydrogen peroxide sensing with reduced graphene oxide/ferroferric oxide nanocomposites," Talanta, vol. 89, pp. 417-421, 2012.

[38] R. Cui, Z. Han, and J.-J. Zhu, "Helical carbon nanotubes: intrinsic peroxidase catalytic activity and its application for biocatalysis and biosensing," Chemistry-A European Journal, vol. 17, no. 34, pp. 9377-9384, 2011.

[39] M. Liu, H. Zhao, S. Chen, H. Yu, and X. Quan, "Stimuliresponsive peroxidase mimicking at a smart graphene interface," Chemical Communications, vol. 48, no. 56, pp. 7055-7057, 2012.

[40] S. Liu, J. Tian, L. Wang, Y. Luo, and X. Sun, "A general strategy for the production of photoluminescent carbon nitride dots from organic amines and their application as novel peroxidaselike catalysts for colorimetric detection of $\mathrm{H}_{2} \mathrm{O}_{2}$ and glucose," RSC Advances, vol. 2, no. 2, pp. 411-413, 2012.

[41] F. Qu, T. Li, and M. Yang, "Colorimetric platform for visual detection of cancer biomarker based on intrinsic peroxidase activity of graphene oxide," Biosensors and Bioelectronics, vol. 26, no. 9, pp. 3927-3931, 2011.

[42] W. Shi, Q. Wang, Y. Long et al., "Carbon nanodots as peroxidase mimetics and their applications to glucose detection," Chemical Communications, vol. 47, no. 23, pp. 6695-6697, 2011.

[43] X. Wang, K. Qu, B. Xu, J. Ren, and X. Qu, "Multicolor luminescent carbon nanoparticles: synthesis, supramolecular assembly with porphyrin, intrinsic peroxidase-like catalytic activity and applications," Nano Research, vol. 4, no. 9, pp. 908920, 2011.

[44] H. Wei and E. Wang, " $\mathrm{Fe}_{3} \mathrm{O}_{4}$ magnetic nanoparticles as peroxidase mimetics and their applications in $\mathrm{H}_{2} \mathrm{O}_{2}$ and glucose detection," Analytical Chemistry, vol. 80, no. 6, pp. 2250-2254, 2008.

[45] Q. Chang, K. Deng, L. Zhu, G. Jiang, C. Yu, and H. Tang, "Determination of hydrogen peroxide with the aid of peroxidase-like 
$\mathrm{Fe}_{3} \mathrm{O}_{4}$ magnetic nanoparticles as the catalyst," Microchimica Acta, vol. 165, no. 3-4, pp. 299-305, 2009.

[46] Z. Jiang, L. Kun, H. Ouyang, A. Liang, and H. Jiang, "A simple and sensitive fluorescence quenching method for the determination of $\mathrm{H}_{2} \mathrm{O}_{2}$ using rhodamine $\mathrm{B}$ and $\mathrm{Fe}_{3} \mathrm{O}_{4}$ nanocatalyst," Journal of Fluorescence, vol. 21, no. 5, pp. 2015-2020, 2011.

[47] Y. Jv, B. Li, and R. Cao, "Positively-charged gold nanoparticles as peroxidiase mimic and their application in hydrogen peroxide and glucose detection," Chemical Communications, vol. 46, no. 42, pp. 8017-8019, 2010.

[48] K. Wang, J.-J. Xu, D.-C. Sun, H. Wei, and X.-H. Xia, "Selective glucose detection based on the concept of electrochemical depletion of electroactive species in diffusion layer," Biosensors and Bioelectronics, vol. 20, no. 7, pp. 1366-1372, 2005.

[49] M. I. Kim, Y. Ye, B. Y. Won, S. Shin, J. Lee, and H. G. Park, "A highly efficient electrochemical biosensing platform by employing conductive nanocomposite entrapping magnetic nanoparticles and oxidase in mesoporous carbon foam," Advanced Functional Materials, vol. 21, no. 15, pp. 2868-2875, 2011.

[50] M. I. Kim, J. Shim, T. Li, J. Lee, and H. G. Park, "Fabrication of nanoporous nanocomposites entrapping $\mathrm{Fe}_{3} \mathrm{O}_{4}$ magnetic nanoparticles and oxidases for colorimetric biosensing," Chemistry - A European Journal, vol. 17, no. 38, pp. 10700-10707, 2011.

[51] M. I. Kim, J. Shim, T. Li et al., "Colorimetric quantification of galactose using a nanostructured multi-catalyst system entrapping galactose oxidase and magnetic nanoparticles as peroxidase mimetics," Analyst, vol. 137, no. 5, pp. 1137-1143, 2012.

[52] M. I. Kim, J. Shim, H. J. Parab, S. C. Shin, J. Lee, and H. G. Park, "A convenient alcohol sensor using one-pot nanocomposite entrapping alcohol oxidase and magnetic nanoparticles as peroxidase mimetics," Journal of Nanoscience and Nanotechnology, vol. 12, no. 7, pp. 5914-5919, 2012.

[53] K. S. Park, M. I. Kim, D.-Y. Cho, and H. G. Park, "Labelfree colorimetric detection of nucleic acids based on targetinduced shielding against the peroxidase-mimicking activity of magnetic nanoparticles," Small, vol. 7, no. 11, pp. 1521-1525, 2011.

[54] J. A. Brinkman, M. Z. Rahmani, W. E. Jones, A. K. Chaturvedi, and M. E. Hagensee, "Optimization of PCR based detection of human papillomavirus DNA from urine specimens," Journal of Clinical Virology, vol. 29, no. 4, pp. 230-240, 2004.

[55] L. Hafner, K. Beagley, and P. Timms, "Chlamydia trachomatis infection: host immune responses and potential vaccines," Mucosal Immunology, vol. 1, no. 2, pp. 116-130, 2008.

[56] M. I. Kim, K. S. Park, and H. G. Park, "Ultrafast colorimetric detection of nucleic acids based on the inhibition of the oxidase activity of cerium oxide nanoparticles," Chemical Communications, vol. 50, no. 67, pp. 9577-9580, 2014.

[57] Z. Zhang, Z. Wang, X. Wang, and X. Yang, "Magnetic nanoparticle-linked colorimetric aptasensor for the detection of thrombin," Sensors and Actuators B: Chemical, vol. 147, no. 2, pp. 428-433, 2010.

[58] B. W. Blais and A. Martinez-Perez, "Detection of group D salmonellae including Salmonella enteritidis in eggs by polymyxin-based enzyme-linked immunosorbent assay," Journal of Food Protection, vol. 71, no. 2, pp. 392-396, 2008.

[59] V. Tripathi, S. Nara, S. K. Chaube et al., "Development of rapid and sensitive one-step direct enzyme linked immunosorbent assay for 17- $\alpha-\mathrm{OH}$-progesterone in serum," Journal of Immunoassay and Immunochemistry, vol. 29, no. 2, pp. 117-127, 2008.
[60] K. D. McReynolds, M. J. Hadd, and J. Gervay-Hague, "Synthesis of biotinylated glycoconjugates and their use in a novel ELISA for direct comparison of HIV-1 gp120 recognition of GalCer and related carbohydrate analogues," Bioconjugate Chemistry, vol. 10, no. 6, pp. 1021-1031, 1999.

[61] H. Hocini, S. Iscaki, J.-P. Bouvet, M. D. Kazatchkine, and L. Bélec, "An ELISA method to measure total and specific human secretory IgA subclasses based on selective degradation by IgA1protease," Journal of Immunological Methods, vol. 235, no. 1-2, pp. 53-60, 2000.

[62] L. Micheli, S. Di Stefano, D. Moscone et al., "Production of antibodies and development of highly sensitive formats of enzyme immunoassay for saxitoxin analysis," Analytical and Bioanalytical Chemistry, vol. 373, no. 8, pp. 678-684, 2002.

[63] L. Gao, J. Wu, S. Lyle, K. Zehr, L. Cao, and D. Gao, "Magnetite nanoparticle-linked immunosorbent assay," Journal of Physical Chemistry C, vol. 112, no. 44, pp. 17357-17361, 2008.

[64] X.-Q. Zhang, S.-W. Gong, Y. Zhang, T. Yang, C.-Y. Wang, and N. $\mathrm{Gu}$, "Prussian blue modified iron oxide magnetic nanoparticles and their high peroxidase-like activity," Journal of Materials Chemistry, vol. 20, no. 24, pp. 5110-5116, 2010.

[65] Z. Tang, H. Wu, Y. Zhang, Z. Li, and Y. Lin, "Enzyme-mimic activity of ferric nano-core residing in ferritin and its biosensing applications," Analytical Chemistry, vol. 83, no. 22, pp. 86118616, 2011.

[66] M.-A. Woo, M. I. Kim, J. H. Jung, K. S. Park, T. S. Seo, and H. G. Park, "A novel colorimetric immunoassay utilizing the peroxidase mimicking activity of magnetic nanoparticles," International Journal of Molecular Sciences, vol. 14, no. 5, pp. 9999-10014, 2013.

[67] V. H. C. Bramwell, G. S. Doig, A. B. Tuck et al., "Changes over time of extracellular domain of HER2 (ECD/HER2) serum levels have prognostic value in metastatic breast cancer," Breast Cancer Research and Treatment, vol. 114, no. 3, pp. 503-511, 2009.

[68] K. S. Asgeirsson, A. Agrawal, C. Allen et al., "Serum epidermal growth factor receptor and HER2 expression in primary and metastatic breast cancer patients," Breast Cancer Research, vol. 9, no. 6, article R75, 2007.

[69] A. Asati, C. Kaittanis, S. Santra, and J. M. Perez, "PH-tunable oxidase-like activity of cerium oxide nanoparticles achieving sensitive fluorigenic detection of cancer biomarkers at neutral pH," Analytical Chemistry, vol. 83, no. 7, pp. 2547-2553, 2011.

[70] K. L. Fan, C. Q. Cao, Y. X. Pan et al., "Magnetoferritin nanoparticles for targeting and visualizing tumour tissues," Nature Nanotechnology, vol. 7, no. 7, pp. 459-464, 2012.

[71] S. Beatty, H.-H. Koh, M. Phil, D. Henson, and M. Boulton, "The role of oxidative stress in the pathogenesis of age-related macular degeneration," Survey of Ophthalmology, vol. 45, no. 2, pp. 115-134, 2000.

[72] C. Korsvik, S. Patil, S. Seal, and W. T. Self, "Superoxide dismutase mimetic properties exhibited by vacancy engineered ceria nanoparticles," Chemical Communications, no. 10, pp. 1056-1058, 2007.

[73] E. G. Heckert, A. S. Karakoti, S. Seal, and W. T. Self, "The role of cerium redox state in the SOD mimetic activity of nanoceria," Biomaterials, vol. 29, no. 18, pp. 2705-2709, 2008.

[74] J. Chen, S. Patil, S. Seal, and J. F. McGinnis, "Rare earth nanoparticles prevent retinal degeneration induced by intracellular peroxides," Nature Nanotechnology, vol. 1, no. 2, pp. 142150, 2006. 
[75] S. M. Hirst, A. S. Karakoti, R. D. Tyler, N. Sriranganathan, S. Seal, and C. M. Reilly, "Anti-inflammatory properties of cerium oxide nanoparticles," Small, vol. 5, no. 24, pp. 2848-2856, 2009.

[76] D.-M. Huang, J.-K. Hsiao, Y.-C. Chen et al., "The promotion of human mesenchymal stem cell proliferation by superparamagnetic iron oxide nanoparticles," Biomaterials, vol. 30, no. 22, pp. 3645-3651, 2009.

[77] N. Ding, N. Yan, C. Ren, and X. Chen, "Colorimetric determination of melamine in dairy products by $\mathrm{Fe}_{3} \mathrm{O}_{4}$ Magnetic nanoparticles- $\mathrm{H}_{2} \mathrm{O}_{2}$-ABTS detection system," Analytical Chemistry, vol. 82, no. 13, pp. 5897-5899, 2010.

[78] J. Zhang, J. Zhuang, L. Gao et al., "Decomposing phenol by the hidden talent of ferromagnetic nanoparticles," Chemosphere, vol. 73, no. 9, pp. 1524-1528, 2008.

[79] J. Z. Jiang, J. Zou, L. H. Zhu, L. Huang, H. Jiang, and Y. Zhang, "Degradation of methylene blue with $\mathrm{H}_{2} \mathrm{O}_{2}$ activated by peroxidase-like $\mathrm{Fe}_{3} \mathrm{O}_{4}$ magnetic nanoparticles," Journal of Nanoscience and Nanotechnology, vol. 11, no. 6, pp. 4793-4799, 2011.

[80] L. Gao, K. M. Giglio, J. L. Nelson, H. Sondermann, and A. J. Travis, "Ferromagnetic nanoparticles with peroxidase-like activity enhance the cleavage of biological macromolecules for biofilm elimination," Nanoscale, vol. 6, no. 5, pp. 2588-2593, 2014.

[81] K. Vickery, A. Pajkos, and Y. Cossart, "Removal of biofilm from endoscopes: evaluation of detergent efficiency," American Journal of Infection Control, vol. 32, no. 3, pp. 170-176, 2004. 

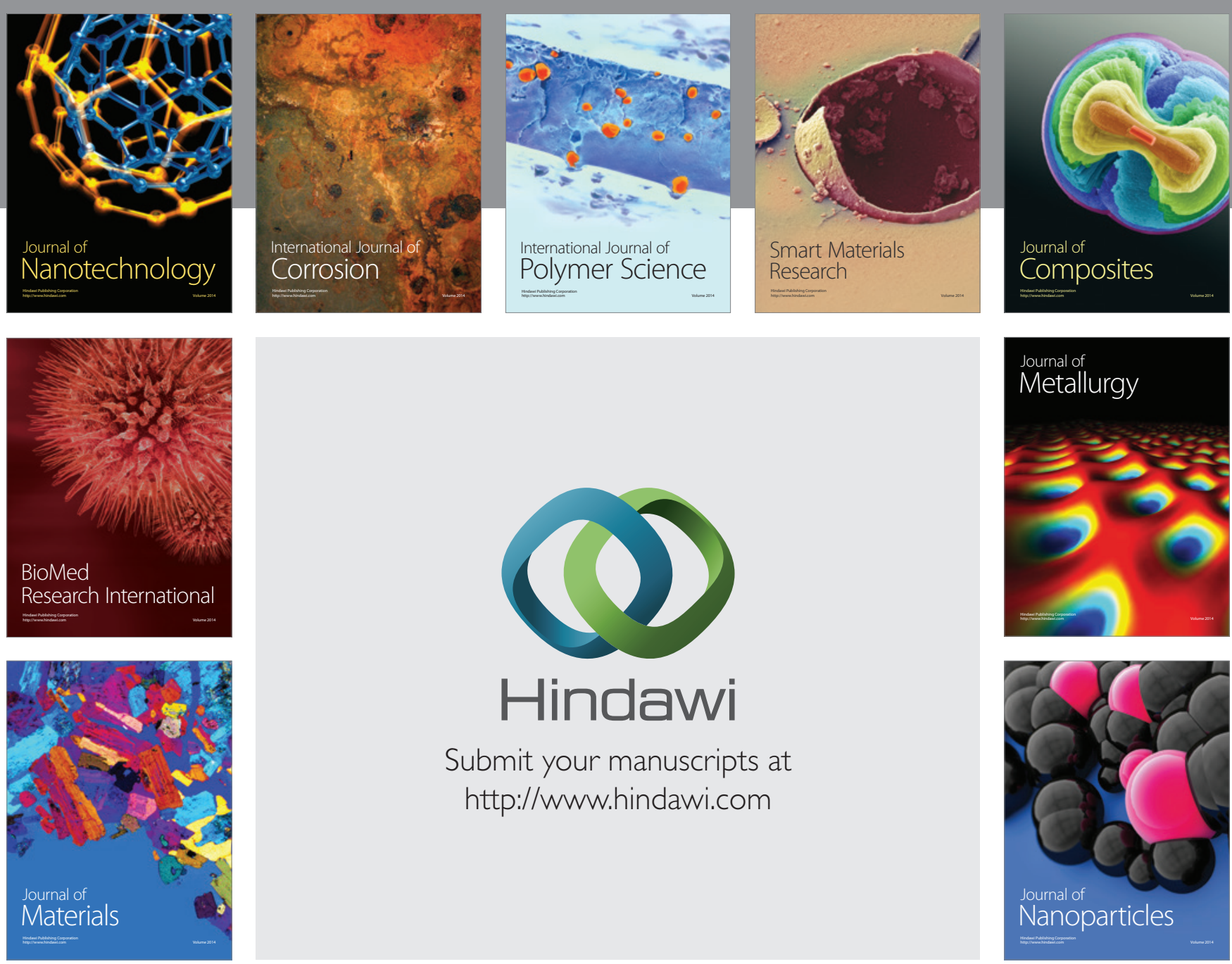

Submit your manuscripts at http://www.hindawi.com
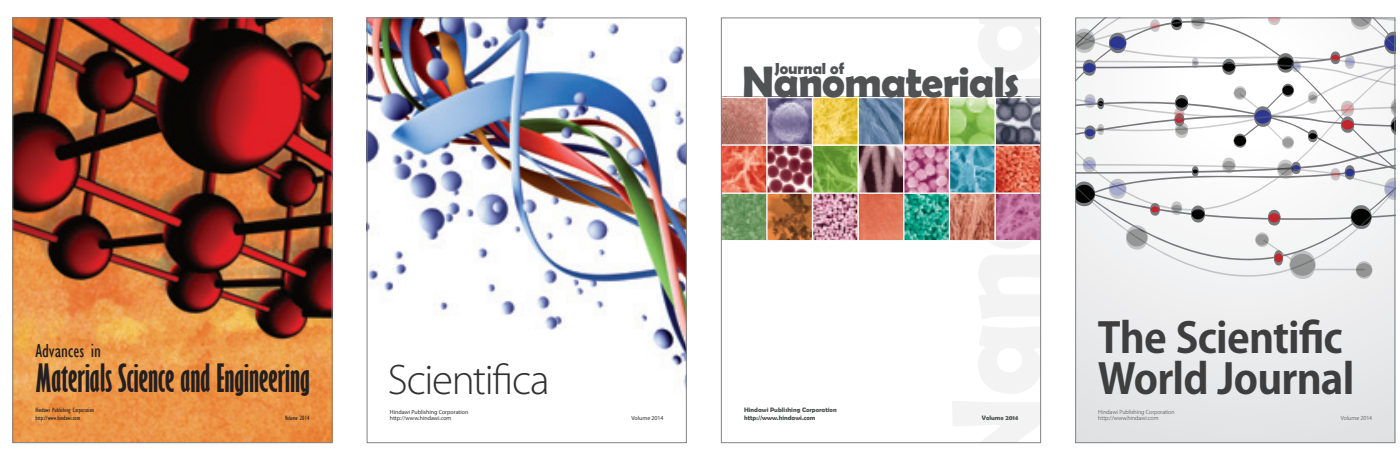

\section{The Scientific World Journal}
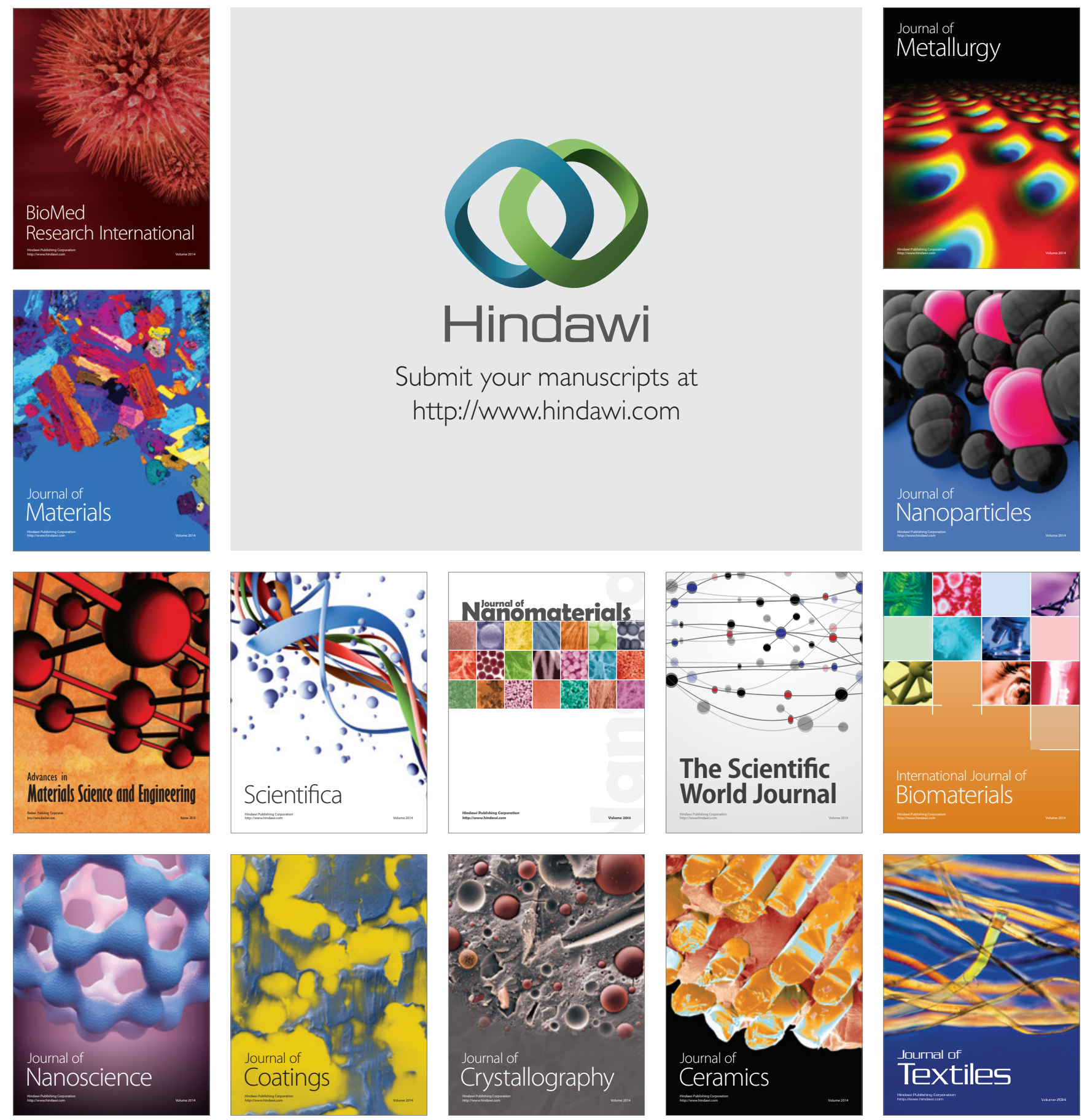